\title{
Proton Therapy for Skull-Base Chordomas and Chondrosarcomas: Initial Results From the Beaumont Proton Therapy Center
}

\author{
Jacob S. Parzen ${ }^{1}$, Xiaoqiang Li $^{1}$, Weili Zheng ${ }^{1}$, Xuanfeng Ding ${ }^{1}$, Peyman Kabolizadeh ${ }^{1}$ \\ 1. Department of Radiation Oncology, Beaumont Proton Therapy Center, Oakland University William Beaumont \\ School of Medicine, Royal Oak, USA
}

Corresponding author: Peyman Kabolizadeh, peymanmcv@gmail.com

\section{Abstract}

Background: Skull-base chordomas and chondrosarcomas are rare tumors that arise directly adjacent to important critical structures. Appropriate management consists of maximal safe resection followed by postoperative dose-escalated radiation therapy. Proton beam therapy is often employed in this context to maximize the sparing of organs at risk, such as the brainstem and optic apparatus.

Methods: This is a single-institutional experience treating skull-base chordomas and chondrosarcomas with postoperative pencil beam scanning proton therapy. We employed a simultaneous integrated boost to the gross tumor volume (GTV) for increased conformality. Demographic, clinicopathologic, toxicity, and dosimetry information were collected. Toxicity was assessed according to Common Terminology Criteria for Adverse Events (CTCAE), v. 4.0.

Results: Between 2017 and 2020, 13 patients were treated with postoperative proton therapy. There were 10 patients with chordoma (77\%) and three with chondrosarcoma (23\%). A gross total resection was achieved in six (60\%) patients with chordoma and one patient with chondrosarcoma (33\%). Nine patients (69\%) received postoperative therapy, whereas four $(31 \%)$ received treatment at recurrence/progression following reexcision. The median dose to the GTV was 72.4 cobalt-Gray equivalents (range, 70.0 to 75.8 ). The mean GTV was $3.4 \mathrm{cc}$ (range, $0.2-38.7$ ). There were no grade 3 or greater toxicities. One patient developed grade 2 temporal lobe necrosis. At 10.7 months' median follow-up (range, 2.1-30.6), the rates of local control and overall survival were $100 \%$.

Conclusions: Proton beam therapy with pencil beam scanning and simultaneous integrated boost to the GTV affords excellent early local control with the suggestion of low morbidity. This method deserves consideration as an optimal method for limiting dose to adjacent organs at risk and delivering clinically effective doses to the treatment volume.

Review began 05/13/2021 Review ended 05/27/2021 Published 05/27/2021

๑) Copyright 2021

Parzen et al. This is an open access article distributed under the terms of the Creative Commons Attribution License CC-BY 4.0., which permits unrestricted use, distribution, and reproduction in any medium, provided the original author and source are credited.
Categories: Radiation Oncology, Neurosurgery

Keywords: proton beam therapy, skull-base chordomas, skull-base chondrosarcomas, pencil beam scanning, simultaneous integrated boost

\section{Introduction}

Skull-base chordomas and chondrosarcomas are rare tumors that are therapeutically challenging due to their proximity to the brainstem, optic apparatus, and temporal lobes of the brain. Owing to low rates of metastases, patients typically succumb to local progression [1,2]. As a result, local therapies are of paramount importance. In general, treatment consists of maximal safe surgical resection followed by postoperative radiotherapy. Unfortunately, gross total resection is not always possible [3]. This may be of particular detriment to patients with chordomas, for whom gross total resection is of relatively greater importance. Historically, photon-based therapies have been utilized, but the inability to achieve therapeutic dosing while respecting normal tissue constraints resulted in inferior clinical outcomes $[4,5]$. To achieve dose $>70$ cobalt-gray equivalents (CGE), proton beam therapy (PBT) is now often utilized at specialized centers offering this approach [6] and results in local control above $90 \%$ for chondrosarcomas and $60-80 \%$ for chordomas [7-9].

To date, much of the published literature has evaluated the use of passive scatter protons [10]. Notable experiences from Massachusetts General Hospital (MGH) and Loma Linda University have outlined initial treatment to a low-risk volume followed by a sequential cone-down to a high-risk volume $[7,8]$. There have been limited reports on active scanning or intensity-modulated proton therapy (IMPT) [11,12]. Passive scattering requires field-specific and patient-specific hardware and is more prone to neutron contamination. Active scanning techniques, such as spot or pencil beam, allow for better conformality of the proximal edge 
of the treatment volume and are better equipped to conform to target volumes constrained by critical structures [13]. This is achieved by accumulating mono-energetic pencil-proton beamlets to dynamically "paint" the target volume. As of 2020, virtually all new proton facilities employ active scanning. Since 2017, we have exclusively treated patients with pencil beam scanning (PBS) technique and employed simultaneous integrated boost (SIB) for increased conformality and better sparing of critical structures when compared to a sequential boost to the high-risk volume. This approach avoids overlap and accumulation of dose outside the target volume which presents a limitation to treatment with a sequential cone-down boost [12]. As both chordomas and chondrosarcomas have an estimated $\alpha / \beta$ ratio of 2 [14], the use of SIB also results in a higher biological equivalent dose (BED) compared to $1.8 \mathrm{~Gy}$ fractions, which may also result in improved tumor control based on the linear-quadratic model. Herein, we present preliminary outcomes with this approach, with a focus on acute and late toxicity, dosimetric parameters, local control, and overall survival.

\section{Materials And Methods}

\section{Patients}

Following institutional review board approval, we queried our proton database for all patients undergoing postoperative PBT for skull-base chordomas or chondrosarcomas. In general, patients were also enrolled on REG001-09, a prospective, multi-institutional registry of proton patients. All patients had histologically confirmed disease and were treated with curative intent. Upon magnetic resonance imaging (MRI) at the time of treatment planning, all patients had some degree of residual disease. No patient presented with Ollier's disease or Maffucci syndrome. The patient's tumor characteristics, radiation treatment details, toxicity, and dosimetric information were all collected.

\section{Proton beam radiotherapy}

Patients underwent three-dimensional CT simulation with a helical CT scanner (Philips Brilliance Big Bore, Philips Healthcare System, Cleveland, $\mathrm{OH}$ ). Intravenous iodinated contrast was utilized. Diagnostic and departmental MRI with T1, T2, and T1 fat-suppressed sequences was completed in the treatment position for optimal image fusion and target/organ-at-risk (OAR) delineation. Gross tumor volume (GTV) was defined as macroscopic tumor as identified on CT and MRI scans. Clinical target volume 1 (CTV1) consisted of the preoperative disease extent with coverage of all intermediate-risk subclinical extent of disease per physician discretion which included the surgical bed plus any area at risk. CTV2 consisted of all high-risk subclinical extent of disease including the surgical bed. Both CTV1 and CTV2 included the GTV. All target volume delineation was completed by a fellowship-trained proton radiation oncologist. Strict adherence to the dose constraints in Table 1 was mandated and would not be sacrificed to enhance target coverage. The brainstem center was defined as the brainstem with an isotropic $3 \mathrm{~mm}$ reduction. All patients were treated with IMPT based on the PBS technique via 3D imaging guidance with cone-beam computed tomography (CBCT). Plans were generated using a five-beam combination of left anterior oblique, left posterior oblique, posteroanterior, right anterior oblique, right posterior oblique and/or vertex beams. Planning was completed with RayStation version 6 (RaySearch Laboratories AB, Stockholm, Sweden) and treatment delivered with the IBA ProteusONE beam model (Ion Beam Application SA, Belgium). Monte Carlo algorithms were used for particle dose calculations. Robust optimization parameters were set to $\pm 3.5 \%$ range and $\pm 2 \mathrm{~mm}$ in $\mathrm{x}, \mathrm{y}, \mathrm{z}$ directions for setup uncertainties resulting in 21 ( 21 for optimization, 26 for robust analysis) perturbed total dose scenarios. Robust optimization was conducted with RayStation version 6 . A robust evaluation was completed with in-house scripting. The maximum dose of $0.03 \mathrm{cc}$ of the volume was extracted for all OARs. The mean and standard deviations of the maximum doses for all perturbed dose scenarios for all patients were calculated based on recalculating the doses with $\pm 3.5 \%$ range and $\pm 2 \mathrm{~mm}$ setup shifts including all diagonal directions (total 26 scenarios). All constraints were also evaluated via RayStation dose-volume histogram (DVH) analysis. This two-step verification with both robust optimization and robust evaluation ensured safety while using IMPT. The relative biologic effectiveness (RBE) was set at 1.1 per institutional standard. CGE was proton dose in Gy multiplied by RBE. All patients were intended to be treated with proton therapy alone, though some received a limited number of fractions with backup photon therapy as needed. Toxicity was graded using the National Cancer Institute Common Terminology Criteria for Adverse Events (CTCAE) version 4.0. 


\section{Cureus}

\begin{tabular}{|l|l}
\hline Organ at risk & Dose constraint \\
\hline Brainstem surface & Maximum dose, $0.03 \mathrm{cc:} 65 \mathrm{CGE}$ \\
\hline Brainstem center & Maximum dose, $0.03 \mathrm{cc:} 50 \mathrm{CGE}$ \\
\hline Optic chiasm & Maximum dose, $0.03 \mathrm{cc:} 61 \mathrm{CGE}$ \\
\hline Left optic nerve & Maximum dose, $0.03 \mathrm{cc:} 61 \mathrm{CGE}$ \\
\hline Right optic nerve & Maximum dose, $0.03 \mathrm{cc:} 61 \mathrm{CGE}$ \\
\hline
\end{tabular}

TABLE 1: Institutional dose constraints.

Abbreviations: cc, cubic centimeter; CGE, cobalt-gray equivalent.

\section{Results}

\section{Patient characteristics}

In total, 13 patients were identified, having received treatment between December 2017 and May 2020. Of the three patients with chondrosarcoma, one had grade 2 disease, and two had grade 1 disease. In addition to the presenting symptomatology in Table 2, there was one instance of each of the following: facial pain, nasal congestion, rhinorrhea, photophobia, dizziness, and gait instability. 


\section{Cureus}

Patient Characteristic

Age, years

Sex

Women

Men

Histology

Chordoma

Chondrosarcoma

Surgery

Gross total resection

Subtotal resection

Indication for RT

Postoperative

Progression/recurrence

Brainstem involvement

Yes

No

Optic pathway involvement

Yes

No

Cranial nerve deficit

VI

Endocrine disorder

Comorbidities

Smoking

Hyperlipidemia

Hypertension

Cardiac disease

Presenting symptoms

Headaches
Diplopia
Change visual acuity
Facial weakness
Ptosis
Otalgia

$8(62 \%)$

$5(38 \%)$

$4(31 \%)$

$9(69 \%)$

No. (\%) or Median (Range)

55 (23-74)

$5(38 \%)$

$8(62 \%)$

$10(77 \%)$

$3(23 \%)$

7 (54\%)

$6(46 \%)$

$9(69 \%)$

$4(31 \%)$

$8(62 \%)$

$6(46 \%)$

$3(23 \%)$

$9(69 \%)$

$4(31 \%)$

$4(31 \%)$

$3(23 \%)$

$7(54 \%)$

7 (54\%)

$4(31 \%)$

$2(15 \%)$

$2(15 \%)$

$2(15 \%)$

TABLE 2: Patient characteristics.

Abbreviations: $\mathrm{RT}$, radiation therapy 


\section{Cureus}

\section{Radiation and dosimetry}

All patients were treated with PBS technique and Table 3 depicts treatment characteristics and dosimetry. All patients received 50.4 CGE to the CTV1. The median dose to the CTV2 was 70.2 CGE (range, 50.4-70.2). The median prescribed dose to the GTV was 72.4 CGE (range, 70.0-75.8 CGE). Eleven out of 13 (85\%) patients received an SIB to the GTV. In all cases, strict adherence to the dose constraints in Table 1 was met. Figures $1 A-1 C$, respectively, depict axial, sagittal, and coronal representations of a representative plan in which SIB was utilized to achieve $75.8 \mathrm{CGE}$ to the GTV. Figures $2 A-2 D$ present the robustness analysis of maximum dose $(0.03 \mathrm{cc})$ to each of the brainstem, optic chiasm, left optic nerve, and right optic nerve. Three patients received less than or equal to three fractions with photon-based therapy due to proton machine maintenance. 


\section{Cureus}

Patient Characteristic

Involved site

Cavernous sinus

Cervical spine

Clivus

Ethmoid

Petrous bone

Sphenoid bone

Suprasellar

Nasal cavity/nasopharynx

Fractions

Fraction size, primary, CGE

Fraction size, secondary, CGE

Target dose

CTV1, CGE

CTV2, CGE

GTV, CGE

Target volume

CTV2 volume, cc

GTV volume, cc

Organs-at-risk (nominal evaluation)

Brainstem surface, $0.03 \mathrm{cc}$ CGE

Brainstem center, $0.03 \mathrm{cc}, \mathrm{CGE}$

Optic chiasm, $0.03 \mathrm{cc}, \mathrm{CGE}$

Left optic nerve, $0.03 \mathrm{cc}$, CGE

Right optic nerve, $0.03 \mathrm{cc}, \mathrm{CGE}$

Left temporal lobe, 0.03 cc CGE

Right temporal lobe, $0.03 \mathrm{cc}, \mathrm{CGE}$

Organs-at-risk analysis (robustness evaluation)

Brainstem surface, $0.03 \mathrm{cc}$ CGE

Optic chiasm, 0.03 cc CGE

Left optic nerve, 0.03 cc CGE

Right optic nerve, 0.03 cc CGE
No. or Median (Range)

5

2

13

3

1

11

8

2

39 (35-39)

$2(1.8-2)$

1.8

50.4

$70.2(50.4-70.2)$

72.4 (70.0-75.8)

$26.6(9.7-138.0)$

$3.4(0.2-38.7)$

61.3 (57.6-64.3)

41.9 (20.3-48.6)

56.8 (8.2-59.4)

57.6 (8.5-59.9)

$57.3(9.3-59.2)$

71.6 (70.1-73.6)

71.9 (32.9-78.0)

$62.3(58.3-64.5)$

57.0 (8.9-60.0)

58.1 (8.7-60.3)

$58.2(9.3-60.0)$

TABLE 3: Tumor and treatment characteristics.

Abbreviations: cc, cubic centimeter; CGE, cobalt-gray equivalent; CTV, clinical target volume; GTV, gross tumor volume. 


\section{Cureus}

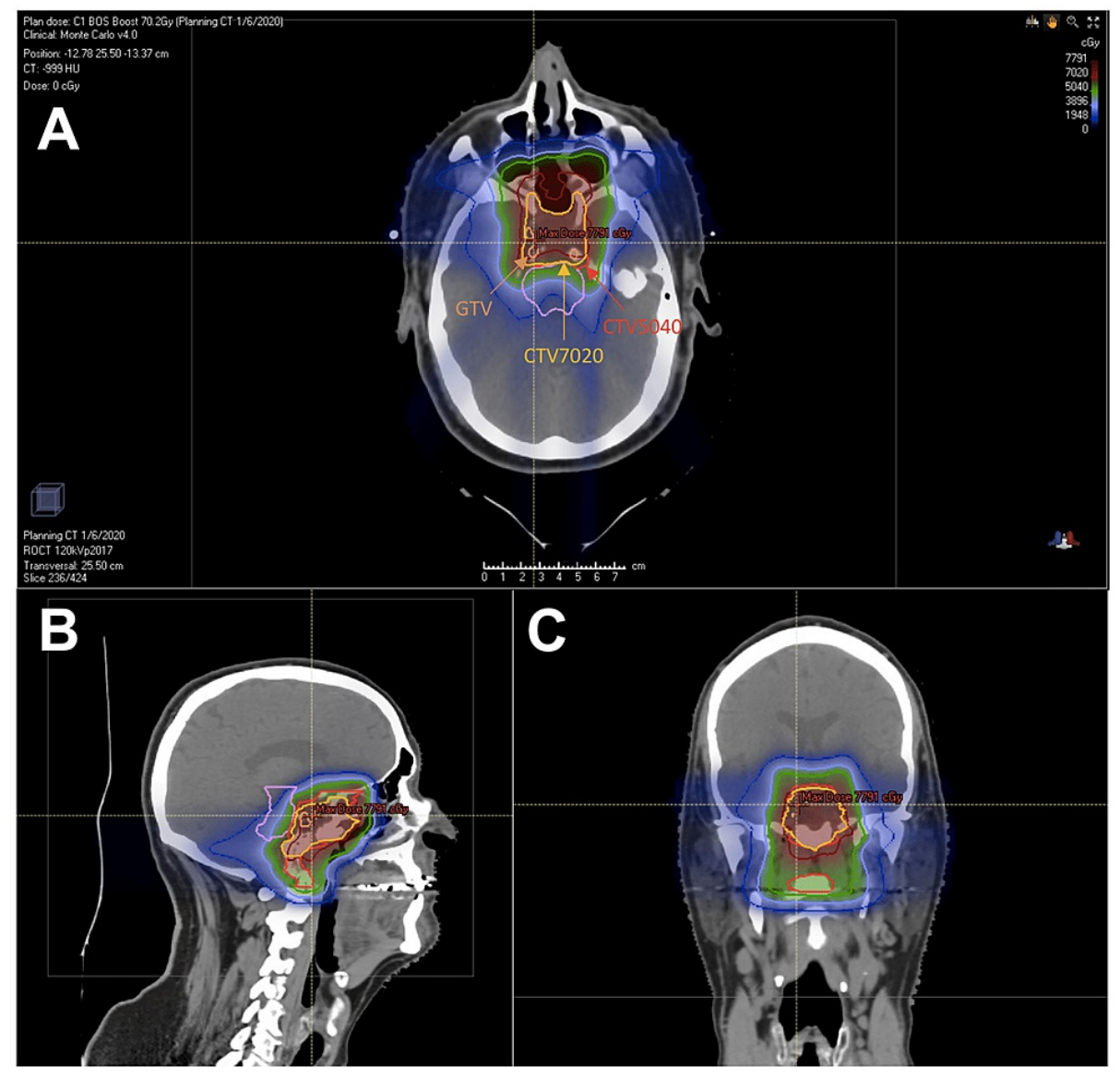

FIGURE 1: Representative (A) axial, (B) sagittal, and (C) coronal images of a patient treated with simultaneous integrated boost to 75.8 cobaltgray equivalents to the gross tumor volume. Pertinent target volumes are denoted on the axial image.

Abbreviations: CTV, clinical target volume; GTV, gross tumor volume. 
A

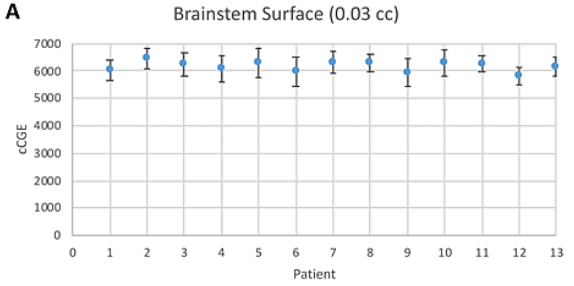

B

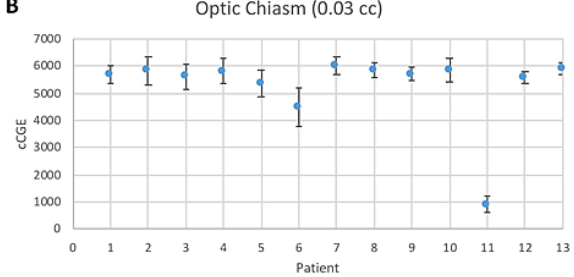

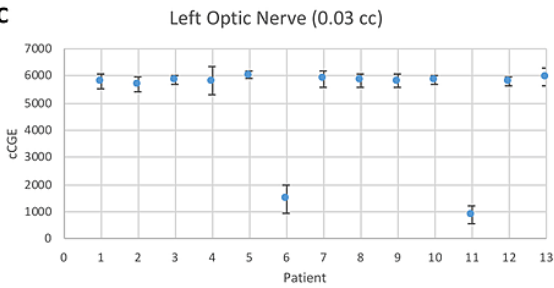

D

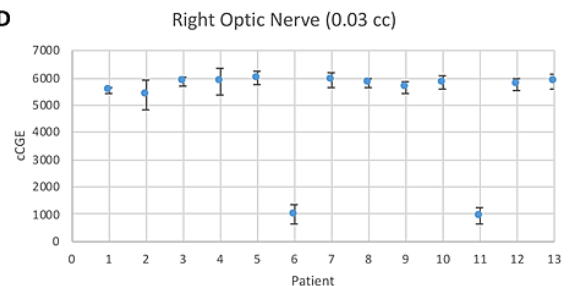

FIGURE 2: Plot of average maximum doses $(0.03 \mathrm{cc})$ to the $(\mathrm{A})$ brainstem surface, (B) optic chiasm, (C) left optic nerve, and (D) right optic nerve for each of the 13 patients with robustness evaluation and error bars representing standard deviations for the $\mathbf{2 6}$ dose scenarios.

Abbreviation: cc, cubic centimeter; cCGE, centi-cobalt-gray equivalent.

\section{Clinical outcomes/toxicity}

With median follow-up of 10.7 months (range, 2.1-30.6), there were no incidences of local or distant failure, and all patients were alive. Three out of 13 patients had at least 24 months of follow-up. There were no episodes of grade 3 or greater toxicity. There were two episodes of grade 1 headache $(2 / 13,15 \%)$, one episode of grade 1 xerostomia (1/13, $8 \%$ ), one episode of grade 1 trismus (1/13, $8 \%$ ), two episodes of grade 1 nausea $(2 / 13,15 \%)$, one episode of grade 1 mucositis $(1 / 13,8 \%)$, and two episodes of grades $1-2$ fatigue $(2 / 13,15 \%)$. One patient developed grade 2 radiation necrosis of the bilateral temporal lobes, which was accompanied by grade 2 fatigue, grade 1 gait disturbance, and grade 2 cognitive disturbance. At last followup, the radiation necrosis had improved with supportive measures. This patient had volume receiving 70 CGE $(D 70)=0.1 \mathrm{cc}$ for the bilateral temporal lobes. For all patients, the median D70 to the left temporal lobe was $0.15 \mathrm{cc}$ (range, 0.0-1.1) and the median D70 to the right temporal lobe was $0.15 \mathrm{cc}$ (range, 0.0-1.6). One additional patient experienced grade 2 mucositis of the soft palate, for which he was re-planned with subsequent resolution of his condition.

\section{Discussion}

Herein, we present our approach using a SIB via PBS to the GTV in patients with skull-base chordomas and chondrosarcomas. With no local failures at median 10.7 months follow-up, this treatment approach has yielded promising early clinical outcomes with no episodes of grade 3 or greater toxicity.

Although histologically distinct, skull-base chondrosarcomas and chordomas share the same management paradigm. In the largest series of proton therapy for skull-base chordomas and chondrosarcomas to date, MGH accumulated 519 patients who were treated from 66 to 83 CGE with a combined photon/proton approach [7]. With a median follow-up of 41 months, local recurrence-free survival was $98 \%$ for chondrosarcomas, $81 \%$ for male chordomas, and $65 \%$ for female chordomas. Importantly, $95 \%$ of failures were local. They employed 1.8 Gy per fraction and incorporated an initial planning volume followed sequentially by one or two cone-down boosts to the intermediate/high-risk regions. This approach has become the widespread standard amongst many institutions offering this specialized treatment. The Loma Linda experience is another notable report [8]. They accumulated 58 patients, treated to a median target dose of 70.7 CGE in 1.8 CGE fractions, with a 94\% three-year local control rate for chondrosarcomas and a $67 \%$ rate for chordomas at a median of 33 months follow-up. More recently, the Paul Scherrer Institute has published the most extensively on treatment with a spot-scanning technique [15]. With chordoma patients receiving a mean dose of 73.5 CGE and chondrosarcoma patients receiving a mean dose of 68.4 CGE, they reported five-year LC rates of $81 \%$ and $94 \%$, respectively, at median follow-up of 38 months. Long-term follow-up has confirmed excellent LC for chondrosarcomas at approximately $90 \%$ [11]. Of note, IMPT was utilized in 20 of the 64 patients in their initial report.

The majority of patients on the current study received an SIB. Investigators at MD Anderson Cancer Center have similarly described the use of SIB using spot either single-field optimized scanning beam plans or 
multifield-optimized IMPT [12]. At median follow-up of 27 months, they reported one local recurrence and one distant failure amongst the 15 patients included in the analysis. Similar to our report, there were no episodes of grade 3 or greater toxicity. In contrast, investigators at Paul Scherrer Institute only used IMPT for sequential boosts. In our experience, the use of SIB simplifies the process of planning and quality assurance through optimization of a single plan with dose painting. The use of $2 \mathrm{~Gy}$ per fraction to the GTV using the SIB PBS technique also results in a higher BED when compared to institutions where 1.8 Gy per fraction is the norm, due to the low $\alpha / \beta$ ratio of 2 for chondrosarcomas and chordomas. The shortened treatment course also theoretically inhibits repopulation of the irradiated tumor cells. We have no local failures at median 10.7 months follow-up, but longer follow-up will be necessary for further evaluation of comparative efficacy. It should be noted that previous reports of extreme hypofractionated approaches, including single-fraction and fractionated stereotactic radiosurgery (SRS), have not yielded acceptable clinical outcomes in chordomas. For instance, the University of Pittsburgh reported a low $53 \%$ five-year tumor control rate for chordomas after a single stereotactic radiosurgery procedure [16]. However, most of the patients in that series had previously undergone multiple resections and fractionated radiation therapy, suggesting a different patient population than the current series. Still, other series have similarly published inferior results for chordomas, though the treatment of chondrosarcomas with SRS may be more reasonable $[17,18]$.

Although our follow-up is somewhat limited, a recent series on chordomas from Florida demonstrated a $14 \%$ rate of local recurrence at two years [19], suggesting that early local failures do occur and lending significant promise to our outcomes thus far. Conversely, a report from the same institution on skull-

base chondrosarcomas demonstrated $100 \%$ local control at two years, with $89 \%$ local control by four years with a median follow-up of 3.7 years [20]. These differences likely reflect the more aggressive local nature of chordomas compared to chondrosarcomas. As 11 of our 13 patients had chordomas, our $100 \%$ local control rate is very promising, but a longer follow-up is needed. We acknowledge that the median GTV at PBT for our patients was $3.7 \mathrm{cc}$, which smaller than other previous series. At Indiana University, the median GTV was $8.1 \mathrm{cc}$, and GTV at the time of radiation therapy as a continuous variable was associated with improved local control [21].

Our cohort has had a very modest toxicity profile. A single patient experienced grade 2 temporal lobe necrosis which has improved with supportive care. The temporal lobe is an important OAR that typically lies in close proximity to the target volume bilaterally. Though no prospectively validated constraints exist, there is suggestion that V70 $>1.7$ cc correlates with increased risk for necrosis [22]. The patient in our series met this constraint, with $\mathrm{V} 70=0.1 \mathrm{cc}$, so there were likely other factors that predisposed them to this toxicity. The MGH experience reported five-year brainstem toxicity of $8 \%$, five-year temporal lobe injury of $8 \%$, and $4.4 \%$ rate of optic neuropathy [7]. Loma Linda reported a $7 \%$ rate of grade 3 or 4 toxicity, including one episode of asymptomatic temporal necrosis [8]. In the previous report of treatment with spot-scanning technique at Paul Scherrer Institute, five-year freedom from high-grade toxicity was 94\% [15]. Importantly, we report no clinical toxicities to the optic apparatus or brainstem, though follow-up is limited.

We acknowledge our small sample size, a result of the rarity of these disease processes. In addition, followup is very limited compared to the pre-eminent series, and this may be clinically meaningful as late failures are a concern, particularly for chordomas. In addition, pathology reports did not include pathological subtyping for chordomas. Hence, we are unable to capture the rate of poorly differentiated chordomas, which are prone to early relapses. At the same time, we present one of the only series to date on PBS for skull-base chordomas and chondrosarcomas. We are one of the first to publish on the use of SIB to the GTV to spare critical structures by avoiding the accumulation of low-dose regions which may affect cumulative dose to OAR. In addition, clinical and toxicity follow-up was performed by our institution, with toxicity prospectively documented by a physician.

\section{Conclusions}

In conclusion, pencil beam proton therapy with SIB is a promising technique to safely deliver dose-escalated treatment in skull-base chordomas and chondrosarcomas. Clinical outcomes and toxicity are very promising with early follow-up and our method merits additional consideration at centers offering this specialized treatment.

\section{Additional Information \\ Disclosures}

Human subjects: Consent was obtained or waived by all participants in this study. Beaumont Health System issued approval 2017-157. Animal subjects: All authors have confirmed that this study did not involve animal subjects or tissue. Conflicts of interest: In compliance with the ICMJE uniform disclosure form, all authors declare the following: Payment/services info: All authors have declared that no financial support was received from any organization for the submitted work. Financial relationships: All authors have declared that they have no financial relationships at present or within the previous three years with any organizations that might have an interest in the submitted work. Other relationships: All authors have declared that there are no other relationships or activities that could appear to have influenced the submitted work. 


\section{References}

1. Forsyth PA, Cascino TL, Shaw EG, Scheithauer BW, O'Fallon JR, Dozier JC, Piepgras DG: Intracranial chordomas: a clinicopathological and prognostic study of 51 cases. J Neurosurg. 1993, 78:741-7. 10.3171/jns.1993.78.5.0741

2. Gay E, Sekhar LN, Rubinstein E, Wright DC, Sen C, Janecka IP, Snyderman CH: Chordomas and chondrosarcomas of the cranial base: results and follow-up of 60 patients. Neurosurgery. 1995, 36:887-96; discussion 896-7. 10.1227/00006123-199505000-00001

3. al-Mefty O, Borba LA: Skull base chordomas: a management challenge. J Neurosurg. 1997, 86:182-9. 10.3171/jns.1997.86.2.0182

4. Zorlu F, Gürkaynak M, Yildiz F, Oge K, Atahan IL: Conventional external radiotherapy in the management of clivus chordomas with overt residual disease. Neurol Sci. 2000, 21:203-7. 10.1007/s100720070077

5. Debus J, Schulz-Ertner D, Schad L, Essig M, Rhein B, Thillmann CO, Wannenmacher M: Stereotactic fractionated radiotherapy for chordomas and chondrosarcomas of the skull base. Int J Radiat Oncol Biol Phys. 2000, 47:591-6. 10.1016/s0360-3016(00)00464-8

6. Palm RF, Oliver DE, Yang GQ, Abuodeh Y, Naghavi AO, Johnstone PAS: The role of dose escalation and proton therapy in perioperative or definitive treatment of chondrosarcoma and chordoma: an analysis of the National Cancer Data Base. Cancer. 2019, 125:642-51. 10.1002/cncr.31958

7. Munzenrider JE, Liebsch NJ: Proton therapy for tumors of the skull base . Strahlenther Onkol. 1999, 175 Suppl 2:57-63. 10.1007/BF03038890

8. Hug EB, Loredo LN, Slater JD, et al.: Proton radiation therapy for chordomas and chondrosarcomas of the skull base. J Neurosurg. 1999, 91:432-9. 10.3171/jns.1999.91.3.0432

9. Feuvret L, Bracci S, Calugaru V, et al.: Efficacy and safety of adjuvant proton therapy combined with surgery for chondrosarcoma of the skull base: a retrospective, population-based study. Int J Radiat Oncol Biol Phys. 2016, 95:312-21. 10.1016/j.ijrobp.2015.12.016

10. Koehler AM, Schneider RJ, Sisterson JM: Flattening of proton dose distributions for large-field radiotherapy Med Phys. 1977, 4:297-301. 10.1118/1.594317

11. Weber DC, Badiyan S, Malyapa R, Albertini F, Bolsi A, Lomax AJ, Schneider R: Long-term outcomes and prognostic factors of skull-base chondrosarcoma patients treated with pencil-beam scanning proton therapy at the Paul Scherrer Institute. Neuro Oncol. 2016, 18:236-43. 10.1093/neuonc/nov154

12. Grosshans DR, Zhu XR, Melancon A, et al.: Spot scanning proton therapy for malignancies of the base of skull: treatment planning, acute toxicities, and preliminary clinical outcomes. Int J Radiat Oncol Biol Phys. 2014, 90:540-6. 10.1016/j.ijrobp.2014.07.005

13. Kanai T, Kawachi K, Kumamoto Y, Ogawa H, Yamada T, Matsuzawa H, Inada T: Spot scanning system for proton radiotherapy. Med Phys. 1980, 7:365-9. 10.1118/1.594693

14. Elsässer T, Krämer M, Scholz M: Accuracy of the local effect model for the prediction of biologic effects of carbon ion beams in vitro and in vivo. Int J Radiat Oncol Biol Phys. 2008, 71:866-72. 10.1016/j.ijrobp.2008.02.037

15. Ares C, Hug EB, Lomax AJ, et al.: Effectiveness and safety of spot scanning proton radiation therapy for chordomas and chondrosarcomas of the skull base: first long-term report. Int J Radiat Oncol Biol Phys. 2009, 75:1111-8. 10.1016/j.ijrobp.2008.12.055

16. Martin JJ, Niranjan A, Kondziolka D, Flickinger JC, Lozanne KA, Lunsford LD: Radiosurgery for chordomas and chondrosarcomas of the skull base. J Neurosurg. 2007, 107:758-64. 10.3171/JNS-07/10/0758

17. Krishnan S, Foote RL, Brown PD, Pollock BE, Link MJ, Garces YI: Radiosurgery for cranial base chordomas and chondrosarcomas. Neurosurgery. 2005, 56:777-84. 10.1227/01.neu.0000156789.10394.f5

18. Hasegawa T, Ishii D, Kida Y, Yoshimoto M, Koike J, Iizuka H: Gamma knife surgery for skull base chordomas and chondrosarcomas. J Neurosurg. 2007, 107:752-7. 10.3171/JNS-07/10/0752

19. Deraniyagala RL, Yeung D, Mendenhall WM, et al.: Proton therapy for skull base chordomas: an outcome study from the University of Florida Proton Therapy Institute. J Neurol Surg B Skull Base. 2014, 75:53-7. 10.1055/s-0033-1354579

20. Holtzman AL, Rotondo RL, Rutenberg MS, et al.: Proton therapy for skull-base chondrosarcoma, a singleinstitution outcomes study. J Neurooncol. 2019, 142:557-63. 10.1007/s11060-019-03129-8

21. McDonald MW, Linton OR, Moore MG, Ting JY, Cohen-Gadol AA, Shah MV: Influence of residual tumor volume and radiation dose coverage in outcomes for clival chordoma. Int J Radiat Oncol Biol Phys. 2016, 95:304-11. 10.1016/j.ijrobp.2015.08.011

22. McDonald MW, Linton OR, Calley CS: Dose-volume relationships associated with temporal lobe radiation necrosis after skull base proton beam therapy. Int J Radiat Oncol Biol Phys. 2015, 91:261-7.

10.1016/i.ijrobp.2014.10.011 